\title{
Comparison Between Xylene And Coconut Oil In Tissue Processing
}

\author{
Dr.Rashmi Chandraker', Dr. Vanita C.Rathod ${ }^{1}$, Dr.Neeraj K. Chandraker ${ }^{2}$, Dr. Siddharth \\ Pundir $^{1}$, Dr.Sudhanshu Dixit ${ }^{1}$, Dr.Veena Desai ${ }^{1}$ \\ 1. Department Of Oral \& Maxillofacial Pathology, RCDSR, Bhilai, India \\ 2. Department of Prosthodontics, GDC, Raipur, India
}

\begin{tabular}{c} 
KEYWORDS \\
Clearing Agent \\
Coconut Oil \\
Histopathology Laboratory \\
\hline Article Info \\
\hline
\end{tabular}

Received 2018/08/15;

Accepted 2018/10/20;

Published Online 2019

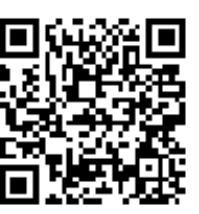

\section{ABSTRACT}

Background: Clearing is an important step in the preparation of histological sections, that removes alcohol and other dehydrants from tissues prior to infiltration of the embedding material (usually paraffin wax).Different types of clearing agents are chloroform, Xylene, Toluene, Paraffin, Methyl benzoate and methyl salicylate \& Citrus fruit oils. The commonly used clearing agent is xylene that is miscible with both alcohol \& parrafin wax. Xylene is supposed to be highly toxic and carcinogenic. As previous research studies have shown the effectiveness of different vegetable oils as clearants, this study was designed to evaluate the efficacy of coconut oil.

Materials and Methods: Two equal halves of 25 oral soft tissue specimens were processed simultaneously in xylene and coconut oil as clearing agents. The Xylenetreated specimens $(\mathrm{XY}-\mathrm{S})$ and Coconut oil-treated specimens $(\mathrm{CO}-\mathrm{S})$ were checked for gross and histological features and comparison was done between the two groups.

Results: Significant shrinkage was noted in $\mathrm{XY}-\mathrm{S}$ compared to that in $\mathrm{CO}-\mathrm{S}$. No difference was found in either of the sections when checked for cellular details and staining quality. Morphometrically, there was significant reduction in the mean cell area in $\mathrm{XY}-\mathrm{S}$ compared to that in $\mathrm{CO}^{-} \mathrm{S}$.

Conclusion: Coconut oil may be substituted for the highly hazardous xylene as a clearing agent without compromising the quality of histological details.

Corresponding Information: Dr.Rashmi Chandraker, Post Graduate Student,Department Of Oral \& Maxillofacial Pathology, RCDSR , Bhilai (Chhattisgarh)

Copyright $\odot$ 2019. This is an open-access article distributed under the terms of the Creative Commons Attribution-noncommercial 4.0 International License which permits copy and redistribute the material just in noncommercial usages, provided the original work is properly cited.

\section{Introduction}

Xylene has been widely used as a dealcoholization agent of choice, inspite of its toxicity to laboratory personnel \& the danger it possess to the environment. The toxic effects of xylene include acute neurotoxicity, cardiac \& kidney injury, cancer, blood dyscariasis, skin disease etc. Various xylene substitutes were used in the past to avoid xylene in the laboratory. However, these substitutes were found to be less effective $\&$ more expensive. Coconut oil is commonly used vegetable oil which is non-toxic, heat stable, slow to oxidize \& has the highest resistance to rancidity.

\section{Aim}

To compare the efficacy of coconut oil \& xylene as a clearing agent in routine histopathological procedures.

\section{Materials \& methods}

Two equal halves of 25 oral soft tissue specimens will be processed simultaneously in xylene \& coconut oil as clearing agent. The duration of clearing was constant for both the processed solutions. The tissue bits were measured before \& after clearing to check for shrinkage. After clearing in xylene \& coconut oil the gross features such as translucency, rigidity and Impregnation was evaluated for each specimen

After Dealcoholization the specimen will also be tested for gross changes \& after clearing all the sections will be stained with Heamotoxylin \& Eosin stain to permit evaluation of the histologic details. 


\section{Evaluation}

1.Gross tissue specimen: After clearing the tissue samples in two different solvents (coconut oil \& xylene), the gross tissue features such as translucency, rigidity (with two fingers), change after impregnation and ease in section cutting were noted down for each specimen separately for coconut oil specimen and xylene specimen.

2.CO-S specimen was less translucent,soft in rigidity \& impregnation compared to XY-S Whereas ease of sectioning was difficult with $\mathrm{CO}$ $\mathrm{S}$ while the quality of staining were found to be same for both CO-S \& XY-S.

3.Cellular architecture:(a) For cellular details, distinct architecture and good nuclear-cytoplasmic contrast is considered.

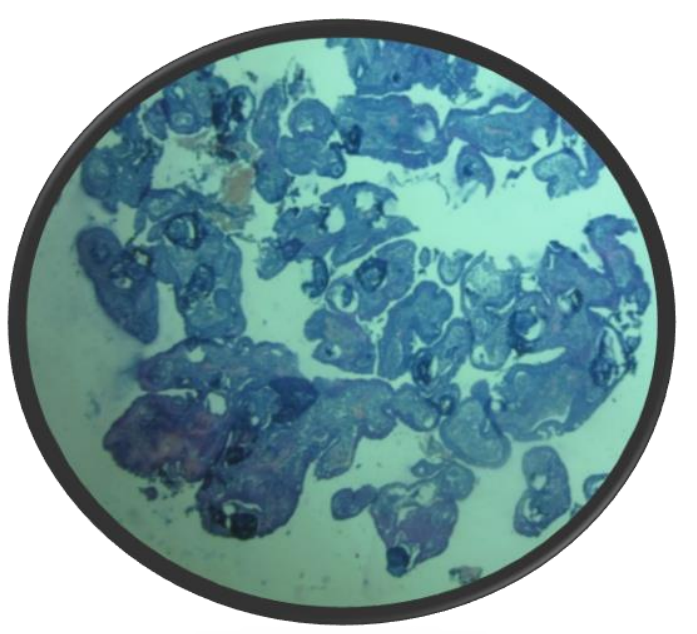

4X-XYLENE SPECIMEN

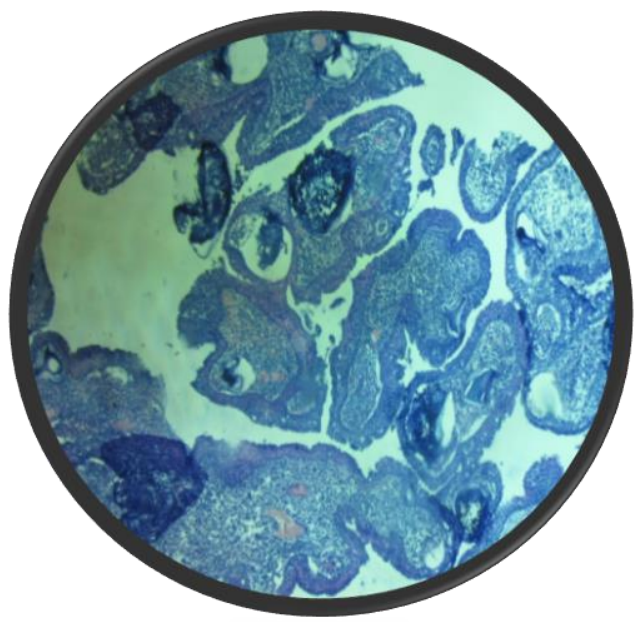

10X-XYLENE SPECIMEN

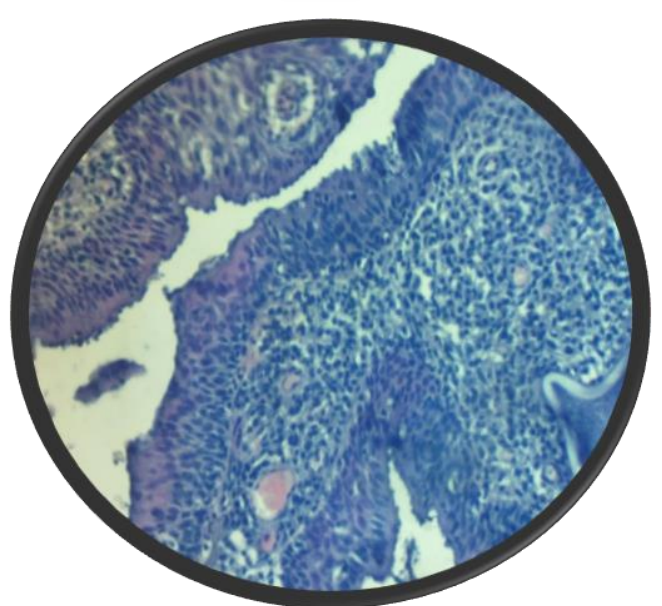

40X-XYLENE SPECIMEN 

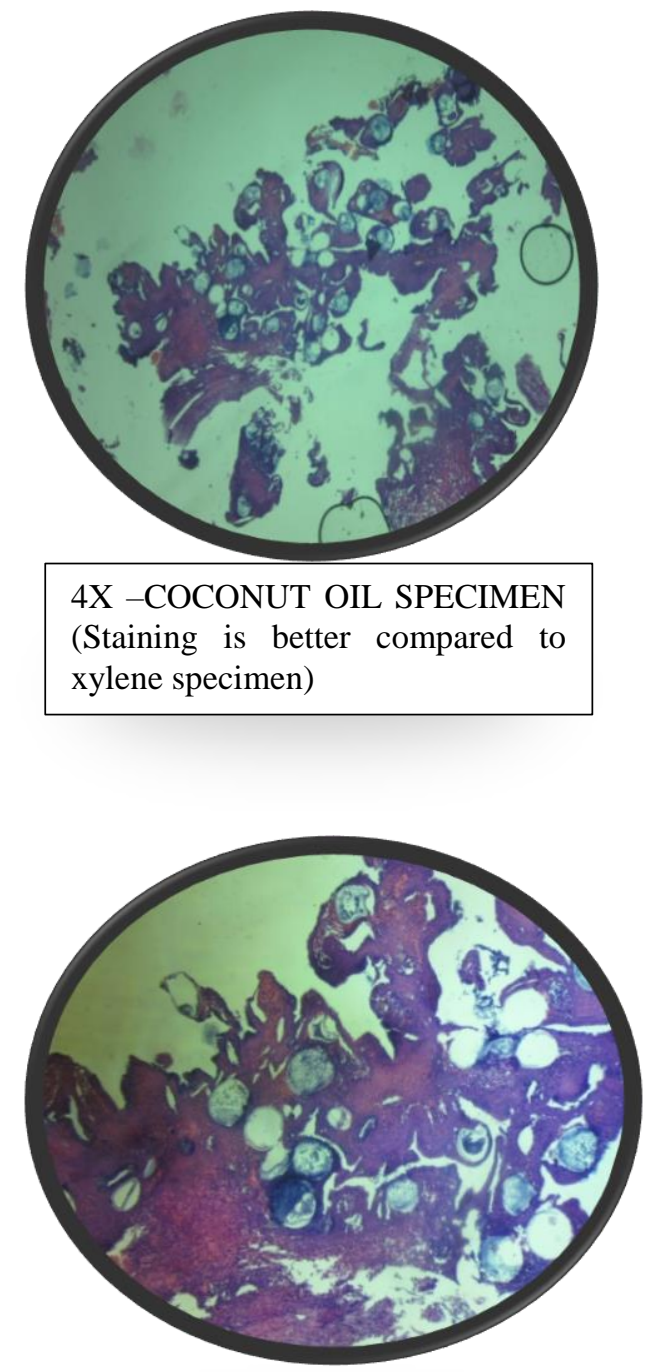

10X-COCONUT SPECIMEN

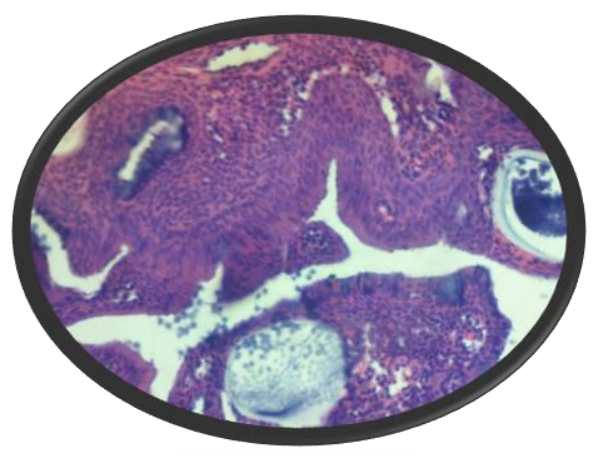

40X-COCONUT SPECIMEN

By both methods staining quality for XY-S \& CO-S were found to be equal.

\section{Results}

\begin{tabular}{|r|c|c|}
\hline Feature & Xylene & Coconut oil \\
\hline Translucency & Translucent & $\begin{array}{c}\text { Less } \\
\text { translucent }\end{array}$ \\
\hline Rigidity & Hard & Soft \\
\hline $\begin{array}{r}\text { Change after } \\
\text { impregnation }\end{array}$ & Hard & Soft \\
\hline Ease of sectioning & Easy & Difficult \\
\hline Quality of staining & Same & Same \\
\hline
\end{tabular}

\section{Discussion}

Considering the toxicity of xylene and its hazards, various substitutes, including vegetable oils and mineral oils, have been tried in the past.

Coconut oil was selected, as it is profusely available in the tropical world, especially in South Asia. Coconut oil or copra oil, is extracted from the kernel or meat of the mature coconuts obtained from the coconut palm (Cocosnucifera). It slowly oxidizes because of its high saturated fat content, and is thus, resistant to rancidification. Coconut oil is a commonly used vegetable oil, available all over the tropical world.

However, most of them showed an inconsistent outcome with vegetable $\&$ mineral oil.

In study done by Sudeendra P et.al in 2014 on 60 soft tissue specimen showed significant shrinkage was noted in xylene sample compared to coconut oil sample, which were similar to our study.

In the present study significant difference was not appreciated in staining in both the sample were as study done by Madhuri et.al in 2011 showed better staining with xylene.

In the study done by Udonkang M et. al on 2014 on 30 soft tissue specimen \& concluded that minor differences between the tissues cleared and dewaxed in bleached palm oil at $600 \mathrm{C}$ and the xylene counterparts in terms of transparency, production of serial sections and quality of histological staining. 
In a study done by Patil S in 2013 conducted a study on 30 embbded paraffin blocks \& concluded that mineral oil was a better clearing agent.

A study by Andre et al.in 1994 substituted xylene with a mixture of peanut oil, soyabean oil, coconut oil and cotton oil and concluded that it was a poor alternative, as the quality of sections with respect to $\mathrm{XY}-\mathrm{S}$ were better. The present study showed sections with similar cellular architecture and better staining quality. Even the special staining procedure showed good results.

As the result of our study showed less shrinkage in CO-S, compared to $\mathrm{XY}-\mathrm{S}$.

During sectioning with microtome difficulty was observed in Coconut oil specimen whereas it was easy in case of Xylene specimen.

The only drawback associated with coconut oil, is its tendency to get solidified at a lower temperature. However, this can be overcome by performing the clearing procedure in an incubator, maintaining the required temperature

\section{CONCLUSION-}

The results of the present study infer that coconut oil is an efficient substitute for xylene, as it is non-hazardous, less expensive and causes less shrinkage of the tissue.

It can be used as a clearing agent in the histopathological laboratory, without losing the quality of the histological details.
Though natural products are cost effective and nonhazardous, the efficiency and commercial availability of chemical products makes them indispensable. Furthermore, studies should be made with an aim to explore more natural products with fixing, clearing, dehydrating and staining properties

\section{Refrences}

1. Kunhua $W$ et al. A Novel Non-Toxic Xylene Substitue (SBO) For Histology. Afr J Tradit Complement Altern Med 2012 ,9 (1);439

2. .Jeyanthi PK et al.Tissue processing of oral biopsy specimens-an adjunct to diagnosis.Journal of Craniomaxillary Diseases 2013, 2(1)

3. Sudeendra P, Acharya s, Javali SB \& Wajid S. Comparing the efficacy of coconut oil and xylene as a clearing agent in the histopathology laboratory. Journal of Oral and Maxillofacial Pathology 2014, 18(1)

4. Andre GG, Wenger JB, Rebolloso D, Arrington JB, Mehm WJ. Evaluation of clearing and infiltration mixtures (CIMs) as xylene substitutes for tissue processing. J Histotechnol 1994;17:137-42

5. Udkong $\mathrm{M}$ et al. Bleached Palm Oil As Substitute For Xylene In Histology 2014,8

\section{How to cite this article:}

Chandraker R, C.Rathod V, K. Chandraker N, Pundir S, Dixit S, Desai V. Comparison Between Xylene And Coconut Oil In Tissue Processing. Mod Med Lab J. 2019; 2 (1) :96-99 